\title{
Children's Reactions to Hospitalization and Illness
}

\author{
Humberto Nagera, MD \\ University of Michigan
}

ABSTRACT: The paper describes children's reactions to illness and hospitalizations from a developmental point of view. Taking the latter into account, it becomes easier to understand not only the child's reaction at different ages and the reasons for it, but the nature of the potential damage, that is, where and why it takes place, as well as the resources available to the child that may help, if wisely used, to minimize the potential traumas.

The emotional reactions of children to illness and hospitalization depends on the type and quantity of the stress or tension produced by the illness, by the hospitalization and by the fantasies-either conscious or unconscious - that the child elaborates around both situations. The final outcome is, in any case, influenced by innumerable other variables as well. For example, his age; his internal balance and level of development; his adaptive capacity; his ability to controlwithin reason; the fears and anxieties that are provoked by the illness; the type of hospitalization; and accompanying procedures, either medical or surgical; the attitude and reactions of the parents; the attitude of the hospital's staff; the environmental conditions of the hospital, etc. All these factors can either facilitate or hinder the child's efforts at adaptation.

Thus, we ought to address ourselves first to the effect of the hospitalization and that of the illness itself on the child. Then, we shall identify those influences (social and familial repercussions, etc.) that both events may exercise in the dynamics of the family and in the child's reaction.

Let us start considering children under the age of five. For them,

Dr. Nagera is Professor of Psychiatry and Chief of Youth Services, Department of Psychiatry, University of Michigan at Ann Arbor. He is also a training analyst with the Michigan Psychoanalytic Institute. 
one of the most vulnerable points in the hospitalization is the separation from the family, and particularly the mother. It results in the sequence described by Robertson [1], Bowlby [2], and others of one first phase of protest followed by one of depression which are, of course, much more easily observable in the young child. In contrast, for the child between the ages of five and ten the significant factor is the psychological and symbolic significance acquired by the hospitalization, the illness, and the treatment. For him, these are the sources of danger, just as the separation from the parents, and particularly the mother, was for the younger child.

For the hospitalized child, the fears, anticipations, fantasies, conflicts, and distortions centered around the hospitalization and illness are important, not only as the unavoidable psychological concomitants but because they can directly influence and prolong the illness itself [3]. Consider for example that a highly excited and frightened child before a surgical procedure may well need larger quantities of sedatives and anesthetics with the concomitant danger that such doses imply. Further, present day experience shows that hospitalizationsespecially when certain types of precautions are not taken-can precipitate or lead in the here and now to various types of emotional problems in some children. They can influence as well the future emotional development and the personality of the child. Many factors can contribute to this outcome such as the separation from home and parents; surgical procedures; medical procedures of a traumatic, painful nature (especially when they are repeated and the child has not had appropriate preparation for it); chronic illnesses of various types; the immobilization that is required in the case of some illnesses and procedures; the danger of death; the hospital visiting rules; the attitude and education of the hospital staff (M.D.'s, nurses, etc.) in relation to the emotional needs of children of various ages; the age of the child; the specific stage of development of the child in the various areas of his personality at that particular point in time; the resources available to him, for example on the side of the ego, such as the degree or ability that he has reached in terms of comprehension; the previous experiences in the life of the child (to include life in general and other contacts with doctors and hospitals in particular); the type of mother-child relationship; the general health and dynamic state of the family; the general emotional health of the family, etc. This list is really incomplete and not intended to cover all the possible contributory factors, but at least it highlights the complexity of the subject.

In any case, what I want to emphasize is the importance of exam- 
ining all of these variables from the point of view of the level of emotional development reached by any given child patient. Thus, separations resulting from hospitalizations are one leading cause of later disturbances. Yet, this is dependent on the age of the child and on the level of development that he has reached. The infant up to the age of two suffers intensely from "separation anxiety" because he is an integral part of a system, of a biological unit, formed by him and his mother. To this genetically determined biological unit are soon added psychological ties of an intense and primitive nature as the infant develops. It is for this reason that the separation of the child from his mother so early in life results in what can best be described as a biological type of vital anguish. (This anxiety, in my opinion, had survival value and developed presumably through millions of years in the process of evolution of the human specie. Incidentally, this same separation-anxiety is clearly visible in many other animal species, and it seems to me possible that the social and cultural superstructure that as humans we have developed may hide its enormous importance.) Separation anxiety reaches its acme some time between the tenth and eighteenth month in the life of the infant, but it is observable up to two and a half years of age. After this it is a much less malignant phenomenon, though still capable of affecting children to different degrees up to the age of five. Between two and a half and five years the child's capacity to tolerate separations from his mother increases markedly. This capacity is enhanced if the separation is well planned and controlled. This increasing ability is due to the advances taking place in his ego development and in the area of object relationships. It allows him to accept, for limited periods of time, maternal substitues when deprived of the maternal objects. (It is for this reason that the age for entry into nursery school is generally around two and a half years or three and rarely before. Children, three and four years of age, under normal conditions would have acquired the capacity to separate from the mother and of accepting maternal substitutes without undue anxiety.) Nevertheless, if the child is ill, running a temperature, in pain, frightened, or hurt, he will lose this capacity. As we know, under such conditions the child's behavior is regressive, and leads to functioning levels much more primitive and appropriate for younger children. This is so partly because the advances in the emotional development of the child, especially in the ego area, are somewhat unstable and tentative to start with. It takes a certain period of time for the mastery of the new skills to be established permanently, so that they are capable of resisting, successfully, the variety of types of stresses to which children are subjected. 
For all the above reasons it is very fortunate indeed that hospitalizations nowadays tend to be much shorter than they were some years back. We have to be grateful for this to progress in medicine and surgery, to the antibiotics, to our present medical ability to prevent certain types of illnesses, such as poliomielitis, etc. In years past, extended separations from the mother during the first year of life resulted, on occasion, in the rather dramatic clinical picture of marasmus. A condition that led children to die slowly and without a logical and apparent reason for it. It was Dr. Spitz in 1945 [4] and 1946 [5] who convincingly showed how the separation from the mother, under certain conditions, led to the clinical pictures that he described as hospitalism and anaclytic depressions. Even more important, Anna Freud [6], Provence, and Lipton [8] and many others have shown, convincingly too, that a child up to the age of eighteen months, if deprived of the presence of his mother-and of the stimulation that the same provides-is a child whose biological maturation is significantly delayed. Thus, for example, he won't be able to hold up his head at three months of age, he won't be capable of sitting at around the sixth month of age, and walking and language are significantly delayed. We know, too, that if this separation is prolonged, irreversible damage to the cognitive and the intellectual function of the child takes place. In fact, it might happen that their IQs will be affected, in the sense that they may never reach the potential with which the child was originally endowed. The stimulation that the mother provides daily during the course of her ministrations to her infant seems to activate biological processes that favor the maturational processes of the brain, that is, the rate of speed of myelinization in the nervous pathways, seems to favor the increase of vascularization in certain areas of the brain, the amount of dendritization that takes place, and so on. In other words, it seems to promote the maturation of the central nervous system in such a way that under ideal conditions the degree of development finally reached approximates the genetic potential with which the child had been endowed. I have referred to these problems in more detail in another publication [7]. I have tried to underline those consequences of the separation through hospitalization in the small child to highlight what might occur beyond the effects that this experience might possibly have in the course of the illness itself, or of surgical procedures, or the difficulties in the management of the child in the hospital ward, or the consequences that this could have for his emotional development or possible later neurosis. The child is thus in the middle of developmental processes that require a "minimum of conditions" so that development can proceed 
in as ideal a form as possible. Interferences with these conditions such as long term separations, especially when poorly planned, can and will affect development on a permanent basis and in negative ways. It should be clear that after the fifth year of life, hospitalizations do not interfere with the processes just described. The separation is not by then the dangerous factor, except in situations that are excessively traumatic and unfavorable.

You would have noticed the close interactions early in life, of the biological and psychological processes, required to mobilize the development of the various aspects of the child's personality. It is essential, then, that the hospitalization of children be considered from all these angles, in order to avoid severe interferences with the child's developmental processes. Or at least, if separations are inevitable to reduce to a minimum possible damage resulting from it. I have referred, too, to the level and quality of the object relationships reached at different ages and to how hospitalizations are capable of interfering with this line of development. A precise knowledge of these various processes is clearly the only way by means of which we are capable of planning a hospitalization intelligently-one that will minimize, or perhaps avoid altogether, the possible negative repercussions that they could have, particularly so at an early age.

It is for this reason that it is essential to have some familiarity with the characteristics of the mental and thought processes of children of different ages. The level of efficiency at which the ego functions at various ages is highly dependent on these factors.

Thus, for example, if we look at the capacity of comprehension that a child has in relation to such things as death and illness, we will realize that they are extremely rudimentary and incomplete early on in life. Concepts such as these are closely associated in everybody's mind (including the child) with hospitals, doctors, and hospitalizations. The concept of death is something that is acquired slowly and progressively during childhood. It is not usual for a child before eleven or twelve years of age to truly understand all the implications and consequences of such a phenomenon. It is partly for this reason that in the child's mind death is such a frightful event. He does not see it as a natural phenomenon but as a punishment, as an enforced separation from those human beings that he is attached to, like an exile from which there is no return either, because God or other omnipotent human beings interfere with the reunion. Death is seen, then, as an active rejection, an active exclusion, because the idea that one is no more is beyond the capacity of comprehending of a child younger than eleven or twelve years of age. 
Exactly the same takes place in relation to the concepts of illness or sickness. The general tendency is to interpret these processes as a punishment, as a sadistic attack on them resulting from their bad behavior, their transgressions of the prohibitions set up by the parents, and so on. This confusion generally persists in children up to eight or ten years. Even then the degree of understanding of which they are capable, though it leads to a better and more realistic grasp of the situation, is still, nevertheless, mixed with fantasies of the type to which we have just referred (punishment, etc.). These fantasies have a universal character and are present in the unconscious mentation of all human beings. This includes adult human beings, who quite frequently tend to interpret, at some level, their own illnesses of those of close relatives as a form of punishment, divine or otherwise. If we add to this that many children have suffered losses of relatives during the course of a hospitalization, or that they may have heard accounts of the "terrible" things that take place in a hospital, we will be able to understand why these fantasies and anxieties proliferate in his mind. To this we must add the tendency of young children to think in very concrete terms. For this reason, they tend to understand in a very literal way all the accounts, real or fictitious, that they may have heard about hospitalizations. They apply such concrete thinking to their own potential experiences there. In this way distortions tend to populate the fantasies of the child. They are sometimes quite surprising in nature. It is these distortions that frequently explain the behavior disorders and the disturbed and anxious manner observable in children while in the hospital, well beyond what is reasonable to expect. In other words, illness, hospitalization and death always have tremendous symbolic meaning and significance, no matter what the present reality of the child's understanding may be like.

The same is true of the role that pain plays. To good measure that role is related to the symbolic and psychological significance that pain, and the fantasies that accompany it, acquire in the mind of the child. Given the special characteristics of the thought processes of children, the child that is in pain, for whatever reason, frequently feels mistreated, persecuted, punished and threatened, as Anna Freud [6] has pointed out. This is a quasi universal norm for children, even in those cases in which the pain is not the result of external manipulations by medical personnel, but the product of the illness itself. This is due to the concretistic and animistic qualities of the thought processes typical for children at certain ages. Freud has also pointed out that when the anxiety arising from the fantasies that accompany 
the pain is minimal, the children have a greater ability to tolerate the pain, and tend to forget the experience quickly. All the above should alert us to the importance of planning a hospitalization very carefully.

Frequently, the negative reactions resulting from illness, medical procedures and hospitalizations are the result of traumas. The traumatic experience may be of major proportions or lesser in significance. In the latter case repetition may lead to a cumulative effect that will act then as a severe trauma. With careful planning, situations of a traumatic character or potentially traumatic can frequently be avoided or at least minimized. But this requires planning and preparation of the child and/or adolescent by means of appropriate information, always given in accordance with the age, stage and degree of development of the child. A traumatic situation is a psychological state during which the ego loses the capacity to keep control over its function, and particularly over the amounts of anxiety that overwhelms it, as well as of the situation that is provoking it. As a result, the ego finds itself totally overwhelmed by anxiety, and either gets paralyzed or takes regressive steps, in terms of its level of function.

The type of reactions or clinical manifestations that are more frequently observable during a period of hospitalization include regressions in behavior and in the child's capacity to function of various types. Regressive behaviors are at the top of the list of observable reactions. For example, the sudden appearance of symptoms such as enuresis and encopresis, or both, in children who had already acquired the capacity to control their sphincters. I mentioned already that ego skills of recent acquisition are the ones abandoned more rapidly. Sometimes, the regression lasts just for a few days, and on other occasions it may be for a period of several weeks, or even months at a time. In the most severe cases it may last for several years, as evidence of the severity of the disturbance triggered off by the hospitalization. The psychiatric treatment of such children is imperative, given the negative repercussions that such regressive symptoms can have, for the future development of their personalities.

Another example is the case of children who have mastered language recently (be that in the form of spoken sentences or just simple words) that may abandon this function, reverting to more infantile types of language. The same is true of the capacity to walk, if it had been acquired recently. A contributing factor to this regression is that hospitalizations tend to place patients in a situation of passivity, infantilizing them to some degree. This is true of both children and adults. They get dressed, bathed and fed. Control is frequently taken 
of their eliminatory functions; special lights may be left on in their rooms, etc. In short, the hospital takes possession of the body of the patient.

Anna Freud [6] has shown that it is not only the adult patient who feels resentful of this invasion. On the contrary, children-and this includes very small children-are similarly resentful of this state of affairs. In the case of children it is the mother who is in charge of the management of their bodies. Suddenly, they find a totally unknown person usurping such a function, that in itself constitutes such an essential element of the very intimate relation between mothers and children. This change is not generally welcomed by the infant. He experiences it like a massive interference in his relationship to his mother, so much so that he may actively resist the ministrations offered by the caretakers of the hospital. This problem is made more serious by the type of functions that many such people will play in the life of the child, including the administration of shots, medicines, etc.

The same situation applies with older children who have the capacity to manage their own body functions independently. These are children who feed themselves, can dress and take baths independently, and can look after their eliminatory functions. The loss of control that such an interference with these functions represents tends to provoke in them marked anxiety. Thus, this type of interference acts like a seductive process, encouraging regressive moves to more infantile levels of functioning, and constitutes a great threat to the ego of those children who have just recently mastered these different skills.

For similar reasons,-nakedness that may be required during medical examinations is very frequently a source of anxiety and resentment. This is particularly so with children in the latency period (5-12 years of age), since they have already acquired strong feelings of shame and modesty, and do not welcome being observed naked by strangers. It is hardly necessary to emphasize that all of these feelings must be taken into account and must be respected during the course of medical examinations and hospitalizations.

Some other forms of regressions observed in small children and infants are the return of thumb sucking (that they may have abandoned years earlier) or in the very young child a renewed interest in bottles (which they may have given up already).

An increase in demandingness, in terms of attention, is quite common. If frustrated this way, we may see them dissolve in tears and despair. Occasionally, these incidents develop into temper tantrums, similar to those typical for the second year of life. The only difference being that it is now happening to a much older child, where you 
don't expect rage to develop in this particular direction, and certainly not to this degree.

It is possible as well to observe behaviors of a highly negative character, tinted by hostile or overtly aggressive acts.

Disturbances of sleep are quite common. For example, difficulties in falling asleep; clinging and demanding behavior, especially requesting the presence of adults; the onset of episodes of nightmares that awaken the child in a state of fright, etc. The content of such nightmares is related to the experiences of the day or of the previous days in the hospital, especially when involving medical or surgical procedures of a painful or frightening nature.

Rapid changes of humor that oscillate between a depressive tone and one of extreme excitement are common. They may be accompanied by unwelcome forms of behavior. This can occur, for example, around feeding time or around a given diet. In the latter case it may become a major management problem for the parents and for the hospital staff. Cases of juvenile diabetes are a notorious and typical example of the difficulties that we are describing.

Tachycardia, palpitations, hyperventilation, diarrhea, etc., are frequent symptoms of children who remain in a state of anxiety while at the hospital.

Hysterical conversion reactions of various types are not uncommon either. They tend to affect the muscles or the sensory organs. It is of interest to note that some of the genuine symptoms of the physical illness are, at a later date, incorporated and/or frequently utilized as models for the hysterical conversion. This happens, for example, with vomiting, aphonia, disturbances of vision, disturbances of walking, and so on. The large majority of these hysterical conversions are of short duration, usually days or just a few weeks. They tend to disappear as soon as the child regains his emotional equilibrium and the excessive tension, due to the illness and the hospitalization, is diminished.

Occasionally, we can observe some types of responses some what more alarming than those mentioned above. I refer to various types of dissociation of the personality, reactive in character. Among them should be mentioned amnesias, and some pseudodelusional conditions. The latter are not always easy to differentiate from genuine delusions due to drugs, high temperatures, and the like.

Surgical interventions always have a significant impact on children. This is dependent, at least in part, on the level of psychological development that the child has reached. Surgical interventions tend to be a nodal point, around which there is a tendency to activate, reacti- 
vate, organize, and rationalize fantasies of a universal character such as that of being attacked, that of being subjugated, damaged physically or castrated. As Anna Freud [6] pointed out, these fantasies are an integral part of the common content (and normal content incidentally) of the mind of children of many different ages. Thus, during the phallic-oedipal phase, the unconscious fear of castration is a typical manifestation of this, not necessarily expressed directly but in symbolic form. The fears of damage to the body, its parts or certain organs are a good example of this. It is for that reason that children between the ages of three and six show an extraordinary interest when they observe, for example, somebody limping, or somebody who has lost an arm, an ear, or a blind person. This interest is accompanied by quite apparent anxiety, and by a multiplicity of questions that are addressed to the parents. Behind all of this is the question, "Can this happen to me?" Surgical interventions are a good vehicle for such fantasies; hence, the special care and preparation required in the case of children who must be subjected to surgical interventions. In this manner, it is possible to avoid much of the potential psychological damage of which they are capable. We should note that such damage as may occur is hardly ever related to the seriousness or severity of the surgical intervention itself, but to the symbolic significance that they have and to the type, intensity and distortions of the fantasies that are associated with them in the child's mind.

You should be aware that similar responses to those that take place in the hospital are possible at home in case of illness. Perhaps they are less frequent and-less intense in nature, since children at home are not overburdened by the anxiety and the complications created by the separation always implicit in a hospitalization. Further, small children-and this includes the large majority of children between four and five years of age-need a certain perceptual constancy. I mean to say that they get easily disorganized in a perceptual milieu which is new to them; in other words, when they are not in those places that are familiar to them, such as home. In the absence of the parents this reaction is facilitated and takes place much quicker.

Another important factor to be considered is that children normally have a rather low threshold for the tolerance of pain, and certainly much lower than that of the adult. This low threshold for pain applies for both physical and psychological pain. This fact must be taken into account by all those professionals handling children in such circumstances.

On the other hand, because the child lacks the necessary ego resources to maintain control when confronted with pain, fear, tension, 
or anxiety, he tends to get disorganized rapidly in their presence. As a consequence of that disorganization, he may produce responses that are maladaptive, negativistic, hostile, and occasionally destructive. We must be cognizant that just as a high temperature is a physiological adaptation to an infectious process, the child produces, too, psychological adaptation in relation to the stress of the hospitalization and the illness. Consequently, many of the symptoms that are observed should be considered at least in some measure as reactive. This is true as well of the negativistic behaviors, tantrums and the like that tend to take place in the pediatric wards, events that are always an irritant to the staff responsible for the welfare of the children, both nurses and physicians alike. It follows that a great deal of tolerance and understanding are absolutely necessary before we try to eliminate, by different means, these so-called undesirable behaviors. As in the case of a high temperature, before we attempt to treat it we must know its etiology, the infectious process responsible for it. In the same manner we must understand the fantasies, conflicts, and anxieties responsible for the undesirable behaviors and symptoms. It is only in this way that we will be in a position to help the child control his excessive anxiety, as well as help him to utilize those means at his disposal with the highest adaptational value.

I should insist that many of the reactions described above are essentially the result of psychological unbalances, generally of a transitory nature. As such they tend to disappear in a few days or a few weeks. Though this is generally true for the large majority of cases, there is a limited number of them where the course of events is quite different. Child psychiatrists and pediatricians know how common it is for parents of children with severe emotional disturbances to ascribe the beginning of the disturbance to an illness or a hospitalization; hence, the importance of the preventive measures.

We will understand as well that such experiences in early childhood are frequently responsible for the unconscious attitudes observed during adulthood towards illness, physicians, and hospitals. It should be obvious that if we are negligent with children, we are probably creating problems that will complicate the professional life of physicians who handle adults. It is said, not without good reason, that the quality of the early experiences in kindergarten have the capacity to destroy our interest in school, and in learning, for many years to come, and occasionally, for the rest of one's life. Similarly, these first experiences with physicians and hospitals will exercise a significant influence in the type and quality of the future patient/physican relationship. Experience shows that many parents who are difficult, and 
sometimes even irrational, when their children are in need of hospitalization or surgical interventions have themselves suffered from negative experiences with the medical progress and hospitals in their own infancies and childhood. Such parents can unconsciously influence in a negative manner the attitude of their children to physicians and to hospitals by passing to the child their own unconscious anxieties. You have to consider that children are part of a social system constituted by the basic family unit. In that system illness, hospitalizations and so on, of necessity, create new dynamic situations capable of influencing the outcome in a positive or negative way. Serious illnesses in children have the potential to create very real difficulties and occasional crisis in the daily handling of the family. These crises are strongly tinted with emotional elements and it is not just parents who are affected but brothers, sisters, grandparents, uncles, aunts, etc.

Family responses are very variable. They need to be studied with care before deciding on the most valuable type of intervention in any particular case. Suffice it to say that the family reaction to the illness or hospitalization is capable of ignoring the emotional needs of the family member affected.

Prugh [7] and Richmond [8] have called our attention to a sequence commonly observed as part of the family's reaction, when in the presence of severe illness in one of the children. They have described a realistic preoccupation at the onset of the illness that is followed quickly by:

1. A phase of negation and incredulity that can last for several weeks or even months.

2. A phase of fear and frustration, usually accompanied by depressive feelings, guilt, recriminations, and marital discord, while the parents try to displace the guilt from one member of the family to the next one, or to the doctor, and so on.

3 . The phase of acquisition of sound information, as well as of intelligent planning. This includes the necessity to learn to live with a certain degree of uncertainty.

The following factors could be of clinical relevance when trying to evaluate the amount of risk involved in any given hospitalization, as well as the possible severity and length of the reactions to be observed.

First, there is the age and level of development of the child. Clearly, the younger the child, the greater the risk.

Second, children who have been previously identified as having psychiatric problems are liable of stronger reactions. 
Third, we should always try to determine if the child has acquired or not the capacity to separate from his mother and the capacity to accept a mother substitute. If this is not the case, the risk is high, and in such cases it would be quite appropriate to allow the mother of the child to accompany him during the hospitalization. It is then easy to conclude that all those hospitalizations, surgical interventions (elective in character), etc., should be postponed, if at all possible, to that time in the life of the child when he would have acquired these skills.

Fourth, it is convenient to explore in general terms how much capacity the child has to express himself verbally, so that he is able to communicate his anxieties, fantasies, and misconceptions. The same should be done in terms of the capacity to react emotionally. I mean the capacity to cry, to verbalize, or to express in any other reasonable form his hostility and anger. The greater the skills the child has in these various areas, the better protected he will be for the experiences that are to come.

Fifth, explore in general terms the child's reactions when facing new situations, especially those that carry with them a certain tenor of anxiety. Naturally, previous hospitalizations and medical manipulations are in this latter group of experiences. In this way, we can form a general idea of what is to be expected, while simultaneously taking any necessary special measures required in order to help and protect those children who tend to react excessively.

Sixth, if a child needs hospitalization, his capacity to relate to other children is an important consideration. It is well-known that misery likes company. In any case, it is surprising the amount of support that children can offer one another.

Seventh, it is important to determine what the balance is in the child's mind between a realistic understanding of his illness, the medical or surgical procedures to be employed, the hospitalization, and the fantasies and misconceptions that are associated with them. This balance must definitely be in favor of reality, at least for those children who have, by reasons of age, the capacity to understand.

Remember that as we have seen already, the most significant part of the damage caused by these different experiences results from fantasies, misperceptions, and misconceptions, and not really by the reality of the situation. In short, the damage is largely due to the subjective interpretation given to these experiences. In order to obtain a good balance, we must rely heavily on parental cooperation so that the necessary information is communicated to the child. Unfortunately, as we have mentioned, it is not all parents who are capable of fulfilling this role. The pediatrician, the surgeon, and if necessary, the 
child psychiatrist will have to contribute more heavily to the preparation of the child in such cases. The type of preparation that is practically possible in most cases consist of:

First, the information to be conveyed should include a description of the procedures and what the child ought to expect. It must be given at a level that is appropriate for his "chronological age," and capacity to understand. The information must conform to reality and be given in a truthful spirit. It should be verbalized in a nonalarming, nontraumatic form. If pain is involved, this should be explained, with a careful description of all the efforts that would be made in order to reduce it to a minimum. It is helpful as well to discuss how the child can contribute to a successful examination or surgical intervention.

Of course, I am well aware that it is always easier to say than to do, a problem that is compounded because children cannot distinguish clearly between suffering that is caused by the illness itself, and that that is caused by the various medical treatments that are utilized during the process of the illness. This is more so at certain ages. Further, since the large majority of children tend to interpret medical manipulations as punishment for their transgressions, disobediences, naughtiness, etc., this element will remain ever present to some degree. This will be so, even in those cases where the reality of the situation has been confronted and explained as extensively and truthful$\mathrm{ly}$ as is possible. Perhaps the most important thing to realize is that we are trying to avoid traumatizing the child, if at all possible, but at the very least trying to minimize it. This can be achieved by offering the child as many opportunities as possible to verbalize and face his fears, his anxieties, his fantasies, and to understand the pain that will be associated with the different procedures required during the hospitalization. Given the many limitations of children at different ages, it is not always possible to do this in an ideal manner.

Second, the information should be offered with reasonable anticipation, so that he can have some time to prepare for it. A period of five to eight days is generally reasonable. The child may pose a number of questions during this time. It is important that he be answered accurately, without distortions or lies. Of course, this ought to be done with sensibility, care and in an appropriate language.

Third, a short visit to the hospital previous to the hospitalization may prove of enormous value.

Fourth, it is essential for pediatric hospitals to have liberal hours for visitation. If necessary, it is desirable to make special arrangements for the mother to stay with the child on a day-to-day, or perhaps even permanent, basis. Nowadays this is a common policy in many such settings and, of course, it is a practice that ought to be encouraged. 
In the preantibiotic era, the fear of possible contaminations or infections was a serious obstacle in this regard.

Fifth, it is important, especially in the case of small children that they be allowed to take some object from the home to the hospital; for example, one or more toys that the child is particularly fond of. Those children who still have a "transitional object" such as a blanket or a teddy bear should be allowed to take it to the hospital. Transitional objects are enormously supportive to the child, facilitating the maintenance of some degree of control over his emotions and fears and helping with his ego functioning in general. The reason for this is that in the child's mind they constitute a symbolic tie of great significance with the home and with the parents.

Sixth, phone calls for those children of an appropriate age are useful and desirable. They contribute to the morale of the child by allowing him to maintain a certain degree of contact with the parents and the home. They help, too, to counteract some of the unconscious fantasies of desertion and abandonment that tend to inhabit the mind of the hospitalized child.

Seventh, it is essential that physicians and nurses take some time to carefully explain to the child those procedures to which he is going to be subjected, and the type and amount of pain that may be involved in the procedures. Naturally, the child should be of an age at which he can have an understanding of what is explained. In any case, this is generally possible even if only in a limited manner. Frequently, physicians and nurses try to hide from the child the procedures to which they are going to subject him. In this way they alarm the child who becomes negativistic, terrified, uncooperative, cries and may become violent. Unfortunately, this type of reaction can be seen on occasion, for a variety of reasons, even when the steps outlined have been followed. Nevertheless, I have no doubt that the appropriate preparation of the child, though it may be somewhat inconvenient for the staff, can significantly reduce, in most cases, the traumatic potential of many of the experiences of the child during the hospitalization. This preparation is indeed essential in cases involving medical procedures that cause pain, or that represent for the child an assault on his physical integrity. But we must accept that in many of these situations there is no ideal manner of handling the problem. We must thus be contented with the lesser evil possible.

It is of some help when at the same time that we explain the situation to the child, we allow him to play with or manipulate some of the instruments that are going to be used in his treatment or medical procedures; for example, the mask in an anesthetic situation. In an attempt to capture his imagination, we can compare such a mask to 
those used by pilots or astronauts. The same is true in relation to stethoscopes, syringes, and the like.

Eighth, in the case of surgical interventions, especially in small children and with children of any age who happen to suffer from severe psychiatric disturbances, we strongly recommend allowing the presence of the mother during the preoperative procedures. It is recommended, too, to allow her to accompany the child to the surgical room. It is important to have the motker present at the moment that the child will wake from the anesthetic. This is a time of great confusion and anxiety and to wake up in pain, and among strangers, is distressing to the child and potentially traumatic.

Ninth, restrictions of the child's movements are frequently necessary. Yet, these procedures should be reduced to whatever is absolutely necessary, both while the child is in the hospital or at home. Restriction of mobility blocks one of the best discharge channels of anger available to children, and particularly so of aggressive tension. Naturally, there are times in which this is unavoidable for orthopedic, surgical, or medical reasons.

When such limitations are imposed-and we all know how difficult it is to enforce it-we frequently observe a marked increase in aggressive outbursts in the behavior of children. This increase in hostility and aggressive manifestations coincides not only with the restrictive period, but sometimes continues itself, once the restriction has been ended. Benign forms of expression of this increase in aggression can be seen in a state of generalized negativism, and in increases in the usage of dirty words, dirty expressions, and offensive language.

Tenth, recreational and activities programs for children of various ages are a great asset to have in a pediatric ward. These programs should be run by professionals with a good awareness of the developmental needs of children and the peculiarities of their psychological processes. Such professionals should be selected on the basis of their ability to motivate children and their ability to earn a child's trust. Play rooms, well-equipped, are essential. These ought to provide toys for children of different ages and with different levels of skills, and should include a fair representation of medical types of toys. The importance of the latter becomes apparent, if we understand that children use toys and play as activities that help them not only in gaining understanding, but in actively mastering many situations that otherwise would lead to anxiety. You will have observed children who, after a frightening visit to a physician, to a dentist, and so on, may adopt in play the role of the professional in relation to siblings, friends, or toys. This type of game, where he pulls teeth or gives shots to other children, tends to repeat itself ad infinitum. This repetition stops at 
the point where the child acquires sufficient mastery over the anxiety provoked by the experience. This type of defense mechanism is very favored by children, and of great service to them. We refer to it as "turning passive into active." Naturally, that is an enormous help when the staff is well acquainted with such defense mechanisms, especially if they are capable of eliciting them. In this way, they can facilitate the process of adaptation to the hospital, as well as the resolution of many of the anxieties of the child. For older children, educational programs are essential. This is particularly important in the case of long-term hospitalization. Such programs help the child to keep his mind occupied in constructive and positive activities and as such away from ruminations and fantasies of a negative or destructive character.

Eleventh, finally, some pediatric hospitals are now experimenting with weekly meetings, organized usually by a social worker or a child psychiatrist. In these meetings the staff nurses, pediatricians, and so on, get together. The purpose is to help children to control the anxieties provoked by the hospitalization, medical procedures, etc. Such meetings have as well a supportive and educative function for nurses and other staff, and as such tend to improve the climate in terms of relations between children and staff. As one of our colleagues, Dr. Poznanski [9] remarks, "This tendency may well become the rule for the future."

\section{References}

1. Robertson J: Young Children in Hospitals. New York: Basic Books, 1958.

2. Bowlby J: Grief and mourning in infancy and early childhood. Psychoanalytic Study Child 7:69, 1952.

3. Blom GE: The reactions of hospitalized children to illness; Pediatrics 22: $590,1958$.

4. Spitz RA: Hospitalism: An inquiry into the genesis of psychiatric conditions in early childhood. Psychoanal Study Child 1:53,1945.

5. Spitz RA: Hospitalism: A follow-up report. Psychoanal Study Child 2:113, 1946.

6. Freud A: The role of bodily illness in the mental life of the child. Psychoanal Study Child 7:69, 1952.

7. Nagera H: Day care centres: Red light, green light, or amber light. Int Rev Psychoanal Vol. 2, Part 1, 121-137, 1975.

8. Provence S, Lipton RC: Infants in Institutions. New York: International University Press, 1962.

9. Prugh DG: Children's reaction to illness, hospitalization, and surgery. The Child 1:181, 1972.

10. Richmond JB: The pediatric patient in illness. In The Psychology of Medical Practice, 1958.

11. Poznanski EO: Special therapeutic considerations. The hospitalized child. Unpublished paper. 